\title{
Um sistema de ventrículo pulmonar produzindo pressão pulsátil em único ventrículo: modelo experimental
}

A pulmonary ventricle system producing pulsatile pressure in single ventricle: experimental model

Bilgein EMRECAN ${ }^{1}$, Mert KESTELLI ${ }^{1}$, Levent YILIK $^{1}$, Banu LAFÇI ${ }^{1}$, Ibrahim ÖZSÖYLER ${ }^{1}$, Ali GÜRBÜZ ${ }^{1}$, Cengiz ÖZBEK ${ }^{1}$

RBCCV 44205-835

\section{Resumo}

Objetivo: Pacientes com ventrículo único funcional têm prognóstico ruim, que resulta em insuficiência cardíaca, mesmo após tratamento cirúrgico. As operações derivação cavo-pulmonar (exceto pelas conexões do átrio direito ao ventrículo direito) não apresentam sistema ventricular pulmonar com pressão pulsátil, além do fluxo reduzido ao ventrículo único. Para resolver o problema, tentamos criar um ventrículo pulmonar que produza pressão pulsátil experimentalmente.

Método: Tentamos criar um sistema ventricular direito que produzisse pressão pulsátil. $O$ modelo experimental foi realizado em seis ovelhas. As pressões do ventrículo pulmonar criado, da artéria pulmonar e do ventrículo esquerdo foram medidas após a saída da circulação extracorpórea.

Resultados: A média das pressões arteriais pulmonares sistólica e diastólica foi 15,6 $\pm 2,0 \mathrm{mmHg}$ e 4,5 $\pm 1,5 \mathrm{mmHg}$. A média da pressão sistólica ventricular esquerda foi 76,6 \pm 4,4 mmHg.

Conclusão: Um ventrículo que produza pressão pulsátil é necessário para a regulação do fluxo da artéria pulmonar, com pressão venosa central e pressão pulmonar não pulsátil nas anomalias como ventrículos únicos funcionais.

Descritores: Ventrículos cardíacos, anormalidades. Cardiopatias congênitas. Derivação cardíaca direita.

1 - Médico, especialista de cirurgia cardiovascular. Hospital de Treinamento e Pesquisa de Ízmir Atatürk, Departamento de Cirurgia Cardiovascular, Ìzmir Turquia.

Trabalho realizado no Hospital de Treinamento e Pesquisa de Izmir Atatürk, Departamento de Cirurgia Cardiovascular, Izmir Turquia.

Endereço para correspondência: 


\section{Abstract}

Objective: Patients with functional single ventricle show bad survey, most of which result in cardiac failure even in the case that they are operated. The right heart bypass operations except for right atrium and right ventricle connections lack pulsatile pulmonary ventricle system besides the volume lack coming to the common ventricle. To solve the problem, we tried to create a pulmonary ventricle which produces pulsatile pressure experimentally.

Methods: We tried to form a right ventricle system which produces pulsatile pressure. The experimental model was carried on six sheep. The pressures of the created pulmonary ventricle, pulmonary artery and the left ventricle were

\section{INTRODUÇÃO}

O termo ventrículo único refere-se às anomalias cardíacas congênitas nas quais há uma única câmara ventricular funcional de onde se originam os vasos de base. Pode ocorrer devido à falha da septação ventricular ou hipoplasia de um dos ventrículos. Outros defeitos cardíacos podem também ter as características do ventrículo funcional único, como a atresia tricúspide. O tratamento cirúrgico consiste de procedimentos paliativos como bandagem da artéria pulmonar e derivações sistêmico-pulmonares. assim como procedimentos definitivos de correção, como aderivação cavo-pulmonar e a septação ventricular. O procedimento de septação é raramente utilizado [1].

Pacientes com um único ventrículo funcional têm prognóstico ruim, que resulta em insuficiência cardíaca, mesmo após serem submetidos ao tratamento cirúrgico. Pacientes submetidos à cirurgia de derivação cavo-pulmonar apresentam índice de 73\% em 15 anos, em condições satisfatórias (excetuando os casos de conexão direta do átrio direito ao ventrículo direito) [2]. As operações de derivação cavo-pulmonar (novamente, exceto pelas conexões do átrio direito e ventrículo direito), não apresentam sistema ventricular pulmonar com regime de pressão pulsátil, além do fluxo reduzido ao ventrículo comum, levando a uma pressão venosa central alta. Para resolver estes problemas, tentamos criar experimentalmente um ventrículo pulmonar que produza pressão pulsátil.

\section{MÉTODO}

Para criar um sistema ventricular pulmonar pulsátil, desenhamos um modelo no qual o fluxo do coração direito é desviado e um sistema ventricular pulmonar é criado para substituir o ventrículo direito (Figura 1). Este modelo foi demonstrado em seis corações de ovelhas com idades entre 1,5 e 2 anos, pesando entre 40 e $45 \mathrm{~kg}$. As ovelhas foram escolhidas para o modelo experimental devido ao fato de measured after leaving the cardiopulmonary bypass.

Results: The mean of the systolic and diastolic pulmonary artery pressures were $15,6 \pm 2.0 \mathrm{mmHg}$ and $4.5 \pm 1.5 \mathrm{mmHg}$. The mean of the left ventricular systolic pressure was mean $76.6 \pm 4.4 \mathrm{mmHg}$.

Conclusion: A ventricle producing pulsatile pressure is necessary for regulating the pulmonary artery flow with high central venous pressure and low non-pulsatile pulmonary pressure in the anomalies with functional single ventricles.

Descriptors: Heart ventricles, abnormalities. Heart defects, congenital. Heart bypass, right.

serem facilmente encontradas no nosso país e também por serem um modelo animal apropriado à pesquisa cardíaca [35]. O estudo foi aprovado pelo Comitê de Ética Institucional. O experimento foi realizado de acordo com o Comitê de Ética para Pesquisas com Animais da Escola de Medicina da Universidade Ege, Ízmir, Turquia. O estudo foi financiado pelos autores. Os animais foram sacrificados quando do término das medidas hemodinâmicas.

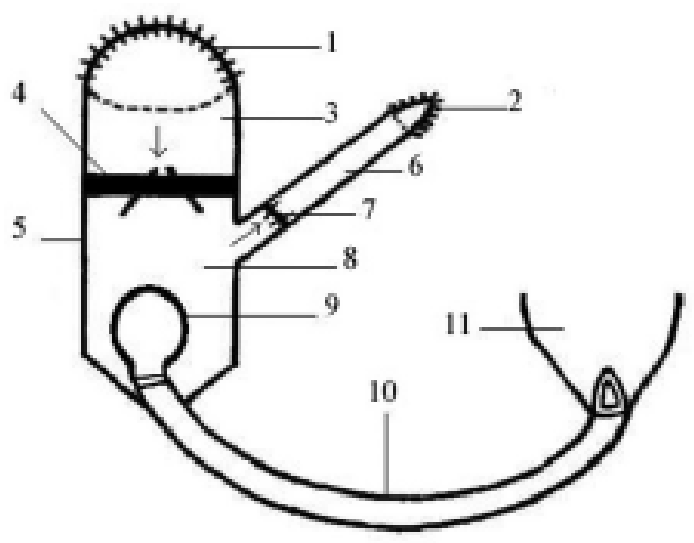

Fig. 1 - Modelo experimental para o ventrículo pulmonar que produz pressão pulsátil. A válvula mecânica de $n^{\circ} 29$ (de forma que ela abrirá $2 / 3$ do enxerto) é colocada em um implante Dacron $30 \mathrm{~mm}$ de $15 \mathrm{~cm}$ de comprimento com sutura contínua de seda 2 0 . Um terço do implante foi anastomosado ao átrio direito do paciente com sutura de propileno 3-0. A conexão da artéria pulmonar do sistema fornecido pelo implante Dacron de $8 \mathrm{~mm}$ no qual havia uma válvula mecânica $n^{\circ} 19$ foi anastomosada ao ventrículo pulmonar criado. 1) Anastomose atrial direita do implante Dacron de $30 \mathrm{~mm}$; 2) Anastomose pulmonar arterial do implante Dacron de $8 \mathrm{~mm}$; 3) Lado direito atrial do sistema; 4) Válvula mecânica de 29 mm; 5) Implante Dacron de $30 \mathrm{~mm}$; 6) Implante Dacron de $8 \mathrm{~mm}$; 7) Válvula mecânica de $19 \mathrm{~mm}$; 8) Ventrículo pulmonar criado; 9) Material elástico em forma de balão que foi fixado na porção final da cânula $n^{\circ} 28$; 10) cânula $n^{\circ}$ 28 ; 11) Ápice ventricular esquerdo. As setas indicam a direção do fluxo 


\section{Técnica cirúrgica}

Seis ovelhas foram submetidas à cirurgia cardíaca com anestesia geral iniciada com xilazina. As ovelhas foram operadas por esternotomia mediana e, após a canulação da aorta, foram realizadas as canulações venosas bicavais. As cavas foram controladas pela aplicação de torniquetes e a operação foi realizada no coração batendo em circulação extracorpórea assistida. A aorta das ovelhas não foi pinçada.

Um material elástico com formato de um balão, cujo diâmetro muda com a pressão, foi fixado na porção final de uma cânula venosa $\mathrm{n}^{\circ} 28$. Quanto esta cânula foi inserida no ápice ventricular esquerdo, observou-se que o balão foi inflado em cada sístole (Figuras 1 e 2).

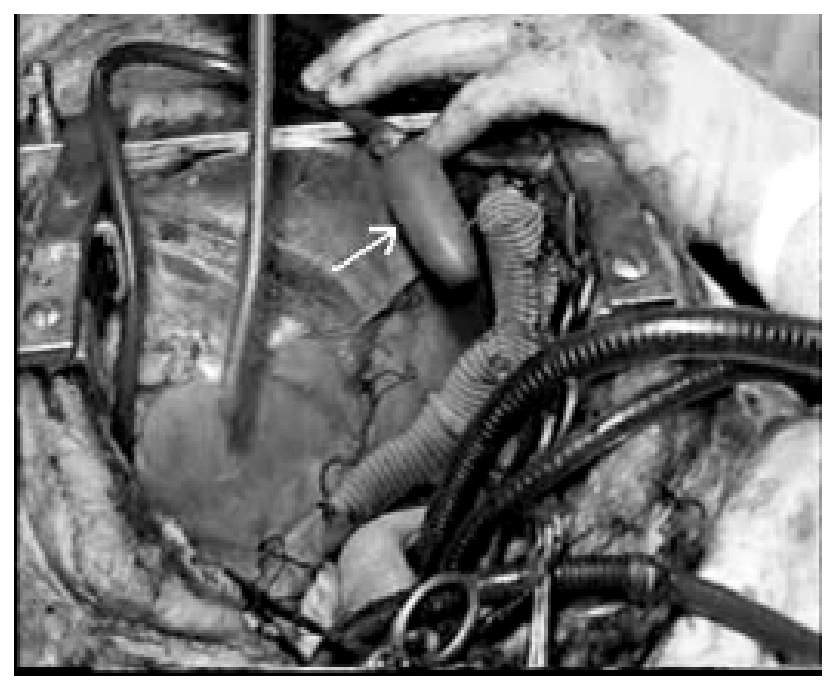

Fig. 2 - Um material elástico (parecido com balão), cujo diâmetro pode mudar de acordo com a pressão, foi fixado à porção final da cânula venosa ${ }^{\circ} 28$. Quando esta cânula foi inserida através do ápice do ventrículo esquerdo, o balão inflava a cada sístole. A seta indica o balão que estava conectado ao ápice ventricular esquerdo através de uma cânula $n^{\circ} 28$

Para criar um ventrículo pulmonar e um átrio direito, uma válvula cardíaca mecânica de 29 mm (de forma que ela pudesse abrir até $2 / 3$ do enxerto) foi colocada num implante Dacron de $30 \mathrm{~mm}$ por $15 \mathrm{~cm}$ de comprimento com sutura contínua de seda 2/0. Um terço do enxerto foi anastomosado ao átrio direito do paciente com sutura de propileno 3/0 (Figuras 1 e 3).

A conexão da artéria pulmonar do sistema mencionado acima foi fornecida por um enxerto Dacron de $8 \mathrm{~mm}$. Uma válvula cardíaca mecânica de $19 \mathrm{~mm}$ foi colocada no implante. O ponto de inserção da válvula no enxerto foi alargado com um retalho de Dacron. Uma extremidade do enxerto foi anastomosada para criar o ventrículo pulmonar e a outra foi anastomosada à artéria pulmonar da ovelha de forma que a válvula permitisse o fluxo para a artéria pulmonar. A parte elástica da cânula venosa $\mathrm{n}^{\circ} 28$, que inflava na sístole e esvaziava na diástole, foi inserida em 2/3 do enxerto (Dacron $30 \mathrm{~mm}$ ) e o implante foi atado à cânula venosa por sutura contínuade fio de seda. Assim, o ventrículo que poderia produzir pressão pulsátil foi obtido (Figuras 1 e 3 ).

Após a conclusão da operação, a circulação extracorpórea foi desligada. Linhas de monitoramento de pressão foram colocadas no ventrículo esquerdo e na artéria pulmonar. Então, a artéria pulmonar foi pinçada e

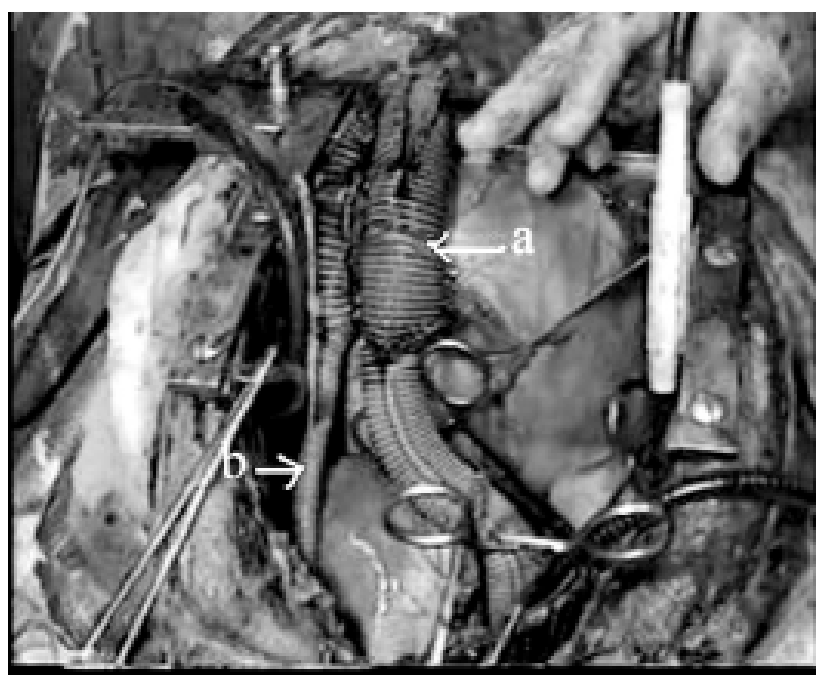

Fig. 3 - Ventrículo pulmonar criado (a). A conexão arterial pulmonar do ventrículo pulmonar criada através de enxerto Dacron de $8 \mathrm{~mm}$ (b)

o desvio do coração direito foi iniciado. Simultaneamente, pressões do ventrículo esquerdo e da artéria pulmonar foram medidas.

\section{RESULTADOS}

As pressões pulmonares sistólicas e diastólicas médias

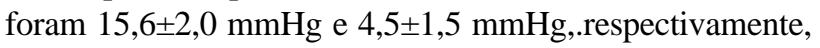
enquanto a média das pressões sistólicas do ventrículo esquerdo foram, em média, 76,6 $44,4 \mathrm{mmHg}$. Estas pressões medidas nas artérias pulmonares das ovelhas foram pulsáteis. 


\section{DISCUSSÃO}

A causa da mortalidade após a operação do desvio de fluxo cardíaco direito (exceto pelas conexões $\mathrm{AD}-\mathrm{VD}$ ) parece ser o fluxo pulmonar não pulsátil e falta de fluxo voltando até o ventrículo que, de fato, está sob aumento crônico do volume. O fluxo sangüíneo pulmonar pulsátil tem um papel importante na regulação da liberação de óxido nítrico do endotélio (mediado pelo shear-stress) e endotelina-1. É também importante na redução da resistência vascular pelo recrutamento capilar passivo. O fluxo não pulsátil em longo prazo conduz à disfunção endotelial e pode estar envolvido na distensão e remodelamento da estrutura vascular devido ao aumento na resistência vascular pulmonar. O fluxo não pulsátil pode levar à formação de shunts artério-venosos, que podem estar parcialmente envolvidos no desenvolvimento de fístulas artério-venosas pulmonares e limitar a atividade física dos pacientes após a operação Fontan [6]. A derivação cavo-pulmonar bidirecional pulsátil parece ser útil aos pacientes de alto risco, não somente na operação de Fontan por etapas, mas também para a paliação definitiva [7].

Portanto, um ventrículo pulmonar que produza pressão pulsátil é necessário para regular o fluxo arterial pulmonar com pressão venosa central alta e pressão pulmonar não pulsátil baixa. Assim, o sistema ventricular mencionado acima poderá ser de grande importância para resolver a falta de volume e fluxo pulmonar não pulsátil. Provavelmente, haveria muitos problemas com a aplicação deste sistema em humanos. No entanto, este modelo experimental mostrou que um ventrículo pulmonar produzindo fluxo pulsátil foi possível no ventrículo único funcional.

\section{CONCLUSÕES}

Como conclusão, a formação de um ventrículo pulmonar que produza fluxo pulsátil pulmonar resolverá problemas encontrados por um fluxo pulmonar não-pulsátil, embora a aplicação deste modelo experimental possa ocasionar outros problemas que, de fato, não são discutidos neste artigo. A aplicação do método em humanos ainda está em estudo.

\section{REFERÊNCIAS}

1. Fontana GP, Permut LC, Laks H. Surgical management of complex functional single ventricle. In: Baue E, Geha AS, Hammond GL, Laks H, Naunheim KS, eds. Glenn's thoracic and cardiovascular surgery. 6th ed. Stanford:Appleton \& Lange;1996. p.1193-201.

2. Kouchhoukos NT, Blackstone EH, Doty BD, Hanley FL, Karp RB. Tricuspid atresia and management of single-ventricle physiology. In: Kirklin JW, Barratt-Boyes BG eds. Cardiac surgery. 3rd ed. Philadelphia:Churchill Livingstone;2003. p.1113-76.

3. Ali ML, Kumar SP, Bjornstad K, Duran CM. The sheep as an animal model for heart valve research. Cardiovasc Surg. 1996;4(4):543-9.

4. Lansac E, Lim HS, Shomura Y, Lim KH, Goetz W, Rice NT et al. Aortic and pulmonary root: are their dynamics similar? Eur J Cardiothorac Surg. 2002;21(2):268-75.

5. Lansac E, Lim HS, Shomura Y, Lim KH, Rice NT, Goetz W et al. A four-dimensional study of the aortic root dynamics. Eur J Cardiothorac Surg. 2002;22(4):497-503.

6. Yin Z, Wang Z, Zhu H, Zhang R, Wang H, Li X. Experimental study of effect of Fontan circuit on pulmonary microcirculation. Asian Cardiovasc Thorac Ann. 2006;14(3):183-8.

7. Miyaji K, Shimada M, Sekiguchi A, Ishizawa A, Isoda T. Usefulness of pulsatile bidirectional cavopulmonary shunt in highrisk Fontan patients. Ann Thorac Surg. 1996;61(3):845-50. 